\title{
Clinical and Demographic Characteristics of WPW Syndrome Attending Arrhythmia Clinic of NICVD \\ M M Rahman ${ }^{1}$, M M R Khan ${ }^{2}$, N Kamal ${ }^{3}$, A K Shamim ${ }^{4}$, M S Akter ${ }^{5}$, M N Uddin ${ }^{6}$
}

\begin{abstract}
:
Wolff-Parkinson-White syndrome is a disorder characterized by presence of an accessory pathway which predisposes patients to tachyarrhythmias and sudden death. Among patients with WPW syndrome, atrioventricular reentrant tachycardia (AVRT) is the most common arrhythmia, accounting for $95 \%$ of re-entrant tachycardias. It has been estimated that one-third of patients with WPW syndrome have atrial fibrillation (AF). $A F$ is a potentially life-threatening arrhythmia. If an accessory pathway has a short anterograde refractory period, then rapid repetitive conduction to the ventricles during $A F$ can result in a rapid ventricular response with subsequent degeneration to ventricular fibrillation (VF). The study population included a total of 255 patients in whom 175 (68.62\%) were men and 80 (31.38\%) were women. Demographic data and clinical characteristics are depicted in Table 1. Left and right WPW syndrome were existing in $70.59 \%$ and $29.41 \%$ of patients respectively. Documented narrow QRS SVT was present in $96.86 \%$, broad QRS SVT was in $2.75 \%$ and AF was in $3.14 \%$ of patients. Antiarrhythmic drugs most frequently used were Digitalis, Metoprolol, Sotalol, Amiodarone and Verapamil.
\end{abstract}

Introduction:

Wolff-Parkinson-White syndrome has attracted cardiologists' attention not only because of its clinical importance but also because of the opportunity it provides to learn about electrical conduction in the heart. ${ }^{1}$ The diagnosis of WPW syndrome is reserved for patients who have both pre-excitation and tachyarrhythmias. Historically, the possibility of the existence of atrioventricular accessory pathways was first raised by Stanley Kent ${ }^{2}$ in 1913. In 1930, Wolff, Parkinson, and White reported on 11 young patients with paroxysms of tachycardia or atrial fibrillation that had a functional

1. Dr. M M Rahman, Department of Cardiology, National Institute of Cardiovascular Diseases (NICVD), Dhaka.

2. Dr. M M R Khan, Department of Cardiology, National Institute of Cardiovascular Diseases (NICVD), Dhaka.

3. Dr. N Kamal, Assistant Professor, Department of Clinical Biochemistry, National Institute of Cardiovascular Diseases (NICVD), Dhaka.

4. Dr. A K Shamim, Department of Cardiology, National Institute of Cardiovascular Diseases (NICVD), Dhaka.

5. MS Akter, Department of Cardiology, National Institute of Cardiovascular Diseases (NICVD), Dhaka.

6. MN Uddin, Assistant Professor, Department of Clinical Biochemistry, National Institute of Cardiovascular Diseases (NICVD), Dhaka.

\section{Corresponding Author:}

Dr. Md. Mahbubur Rahman, Assistant Professor of Cardiology, NICVD, Dhaka. bundle branch block and an abnormally short PR interval on electrocardiograms recorded during sinus rhythm. ${ }^{1}$ In 1933, Holzmann and Scherf ${ }^{3}$ reported that the mechanism in Wolff-Parkinson-White syndrome consisted of an acceleration of passage of the impulse from the atria to the ventricles and not a block, as had been proposed by Wolff, Parkinson, and White. In 1944, Ohnell ${ }^{4}$ introduced the term "preexcitation" to the medical literature.

Among patients with WPW syndrome, atrioventricular reentrant tachycardia (AVRT) is the most common arrhythmia, accounting for $95 \%$ of re-entrant tachycardias. Atrial fibrillation (AF) is a potentially life-threatening arrhythmia in patients with WPW syndrome as it can degenerate to ventricular fibrillation (VF).

\section{Definition of WPW syndrome 5}

Symptoms suggestive of recurrent tachycardia in addition to the following ECG characteristics;

* Shortened P-R interval of $<0.12 \mathrm{~s}$

* Slurred slow rising onset to QRS known as the delta wave

* A prolonged QRS complex $>0.11 \mathrm{~s}$

\section{Population:}

All the patients were presented at arrhythmic clinic at OPD. For all patients the following protocol was followed: detail history, physical examination, 12 lead ECG analysis, and echocardiogram. The 12 lead ECG was used to classify the pathway as left sided or right sided. The documented tachycardias were classified as narrow wave tachycardias, broad QRS tachycardia and atrial fibrillation.

$\begin{array}{lc}\begin{array}{l}\text { Variables } \\ \text { Age (years) }\end{array} & \begin{array}{l}\text { Values } \\ 32 \pm 16(\text { range } 13 \\ \text { Men/women } \\ \text { Presentation }\end{array} \\ \begin{array}{l}\text { Symptomatic } \\ \text { tachycardia }\end{array} & 255(68.62 \%) / 80 \\ \begin{array}{l}\text { Presyncope } \\ \text { Recurrence of }\end{array} & 50(100 \%) \\ \text { after ablation } & 13(5.00 \%) \\ & \\ \text { Table-2: Antiarrhythmic drugs most frequ } \\ & \\ \text { Drugs } & \text { Number (\%) } \\ \text { Digitalis } & 08(3.13 \%) \\ \text { Metoprolol } & 67(26.27 \%) \\ \text { Sotalol } & 23(9.01 \%) \\ \text { Amiodarone } & 12(4.70 \%) \\ \text { Verapamil } & 122(47.84 \%)\end{array}$


Table-3: Associated diseases

Associate diseases

Aortic valve prosthesis

Number of cases

Mitral valve stenosis

$01(0.39 \%)$

$04(1.56 \%)$

$11(4.31 \%)$

$12(4.70 \%)$

$08(3.13 \%)$

$04(1.56 \%)$

$04(1.60 \%)$

Tachycardiomyopathy

Table-4: WPW classification

$\begin{array}{lll}\text { Class } & \text { Number } & \text { \% } \\ \text { Left } & 180 & 70.59 \% \\ \text { Right } & 75 & 29.41 \%\end{array}$

Table-5: Documented arrhythmia

$\begin{array}{lll}\text { Arrhythmia } & \text { Number } & \text { \% } \\ \text { Narrow QRS SVT } & 240 & 94.12 \% \\ \text { Broad QRS SVT } & 07 & 2.75 \% \\ \text { AF } & 08 & 3.14 \%\end{array}$

Statistical Method:

Data were presented as mean value \pm SD. Students t test was applied to see difference between discrete variable. $\mathrm{P}$ value $<0.05$ were considered significant.

\section{A brief history of WPW syndrome:}

Wolff, Parkinson, and White first described the syndrome known as WPW syndrome in 1930 in a report of 11 patients with a short P-R interval, "bundle-branch block," and paroxysmal superventricular tachycardia and/or atrial fibrillation. ${ }^{6}$ It was not until 1959 that accessory AV pathways were determined to be responsible for the findings in WPW syndrome; although such pathways had been described in 1893 by Stanley Kent. ${ }^{7}$ This discovery has allowed electrophysiologists to map out and ablate accessory pathways. The introduction of radiofrequency energy for use in ablative procedures in the late 1980s allowed for tremendous improvements in the success rates of this procedure. In clinical practice today, nearly all accessory pathway locations are amenable to attempts at ablative therapy, with reported recurrence rates between $2 \%$ and $9 \%{ }^{8}$

\section{Results:}

The study population included a total of 255 patients in whom 175 (68.62\%) were men and 80 (31.38\%) were women. Demographic data and clinical characteristics are depicted in Table 1. Left and right WPW syndrome were existing in $70.59 \%$ and $29.41 \%$ of patients respectively. Documented narrow QRS SVT was present in 94.12\%, broad QRS SVT was in $2.75 \%$ and AF was in $3.14 \%$ of patients. Antiarrhythmic drugs most frequently used were Digitalis, Metoprolol, Sotalol, Amiodarone and Verapamil.

\section{Discussion:}

\section{Pathophysiology}

Preexcitation occurs when an atrial impulse is conducted in an anterograde (atria to ventricle) fashion down an AV accessory pathway before the AV node-His- Purkinje axis begins depolarization. How this manifests clinically and on the ECG depends on how much faster the atrial impulse is conducted down the accessory pathway into the ventricular myocardium as opposed to the AV node-His-Purkinje axis. The classic "short P-R" interval description of WPW syndrome is a result of premature ventricular depolarization mediated by the AV accessory pathway. This early ventricular depolarization manifests on the ECG as both a short P-R interval and a slurred upstroke in the $\mathrm{R}$ wave, referred to as the delta wave. The delta wave represents relatively disorganized and slow ventricular depolarization via the accessory AV

pathway and ends at the point at which AV nodal conduction begins to successfully propagate, thus creating

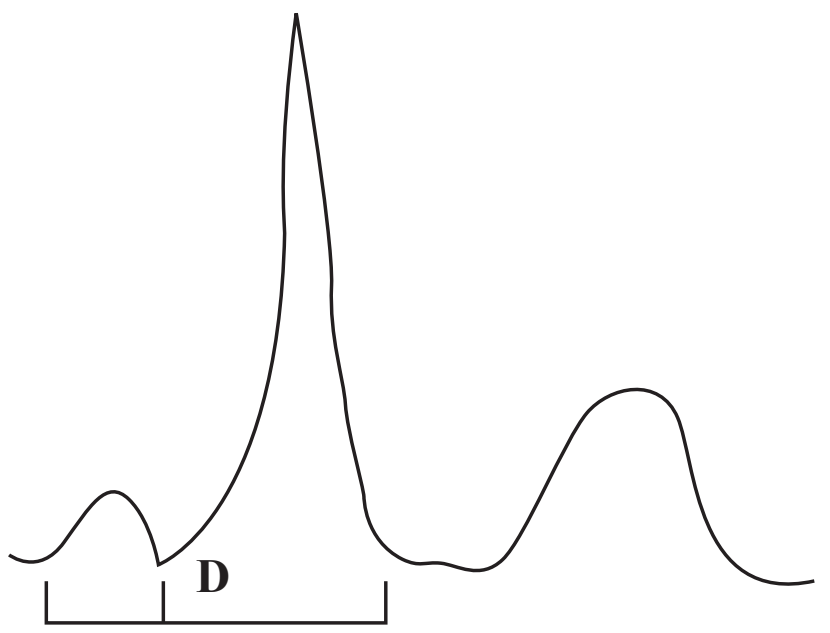

Fig. 1 The impulse formation and conduction process in a patient with WPW syndrome in normal sinus rhythm. "D" the delta wave, is the segment of ventricular myocardium that depolarizes earlier than anticipated had normal conduction occurred.

Other ECG manifestations may include a prolonged QRS complex and secondary ST-T waves changes that are discordant to the QRS vector. The presence of a delta wave indicates that AV accessory pathway conduction is faster than AV node conduction, creating a substrate that can predispose to preexcitation arrhythmias in the setting of either $\mathrm{AV}$ nodal blockade or rapid rates of atrial depolarization, such as atrial fibrillation. Rates of pre-excited tachycardias depend on the length of the refractory period of the accessory pathway tissue, which is usually longer than that of the AV nodal tissue. This difference in refractory period length provides the substrate by which a premature atrial depolarization can incite AV reentry tachycardia (AVRT).

\section{Frequency:}

Delta waves detectable on an ECG have been reported to be present in $0.15 \%$ to $0.25 \%$ of the general population. ${ }^{9}$ A higher prevalence of $0.55 \%$ has been reported in first-degree relatives of patients with accessory pathways. Wolff-Parkinson-White syndrome is more commonly" 
diagnosed in men than in women, although this sex difference is not observed in children. Among those with the Wolff-Parkinson-White syndrome, 3.4 percent have first-degree relatives with preexcitation. ${ }^{10}$

\section{Electrocardiographic features of WPW syndrome}

There are 3 ECG criteria for diagnosing WPW syndrome; a short $(<120$ milliseconds) PR interval, a delta wave, and an anomalous QRS configuration. The last -of these is the most variable factor and depends on the location of the bypass tract. The most common locations for accessory AV pathways are, in decreasing order of frequency, left lateral (free wall), posteroseptal, right free wall, and anteroseptal.
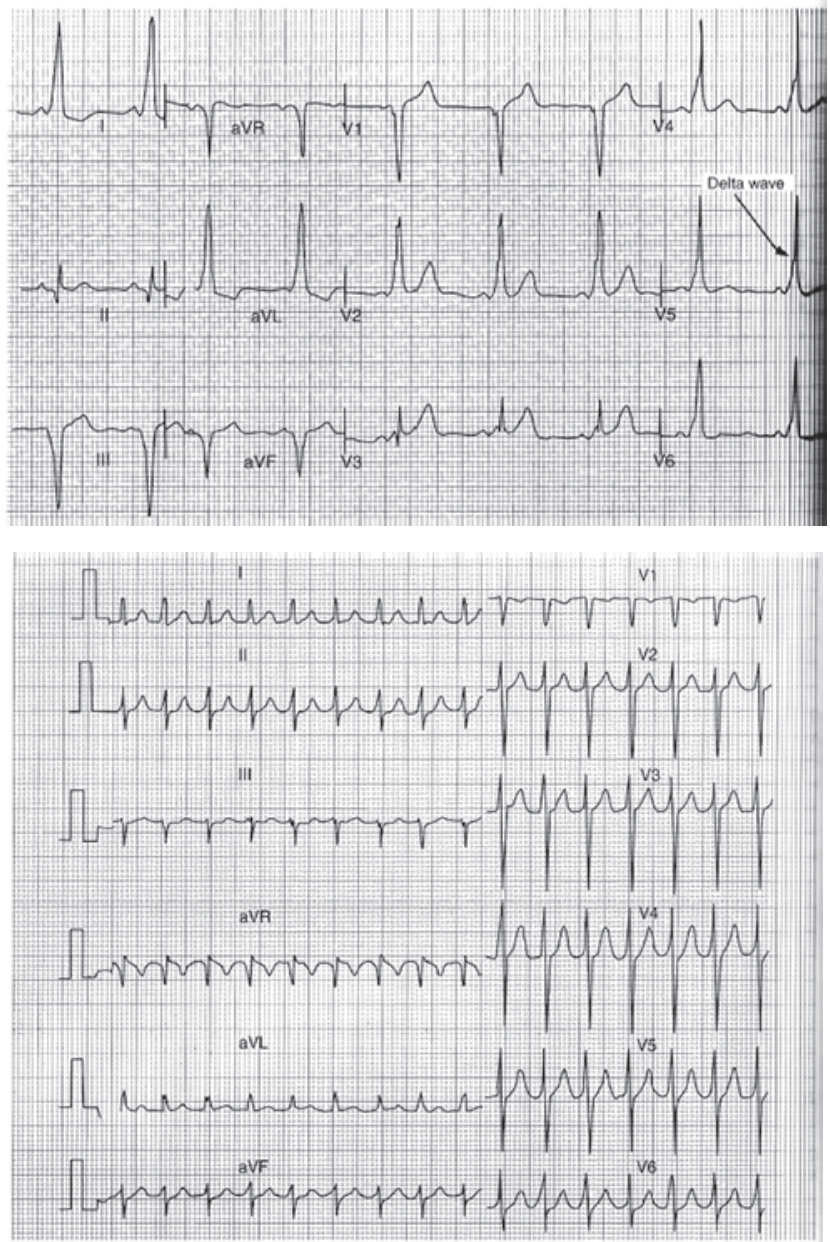

\section{Electrocardiographic features of WPW Syndrome}

Accessory AV pathway activity is determined by the electrophysiological properties of the pathway tissue, including the rate of tissue depolarization, length of the refractory period, and differentials in these properties when confronted with anterograde (atria to ventricle) or retrograde (ventricle to atria) directions of conduction. An accessory AV pathway can be seen as 2 separate tracks, one running from the atria to the ventricle and the other heading in the opposite direction. The clinical syndrome of WPW can likewise be categorized into 2 separate disorders: (1) those arrhythmias that result from anterograde accessory AV pathway conduction (resulting in a wide QRS complex) and (2) those that use retrograde accessory AV pathway conduction (resulting in a narrow QRS complex).

\section{Orthodromic reciprocating tachycardia or AVRT}

ORT, or AVRT, is a common presentation of WPW syndrome, as most accessory AV pathways are in fact only capable of retrograde conduction. Atrioventricular reentry tachycardia can be initiated in the setting of a premature atrial complex when the refractory period of the accessory $\mathrm{AV}$ pathway is longer than that of the AV node. For this to occur, the premature atrial complex must occur early enough in the cardiac cycle to be blocked from anterograde conduction down the accessory pathway but late enough to allow for successful conduction through the AV node. As a result, a normally conducted impulse from the atria is spread throughout the ventricles by the AV node-His-Purkinje axis (referred to as orthodromic conduction) and passes back into the atria in a retrograde fashion though the now excitable accessory AV pathway. As long as the atrial fibers are not refractory to depolarization at the time this impulse arrives, the atria will depolarize prematurely (ie, before the sinus node depolarizes), setting up a reentrant circuit. Rates of ventricular depolarization typically range between 150 and 250 beats per minute. A representative ECG of AVRT is shown. Note that the $\mathrm{p}$ wave is distinctly seen following the QRS, giving a "short r-p interval" tachycardia. This is in contrast to the typical form of $\mathrm{AV}$ nodal reentry tachycardia (AVNRT), in which the $p$ wave is often buried in the QRS complex due to simultaneous activation of both atria and ventricles. However, a discrete $\mathrm{p}$ wave can be appreciated following the QRS complex in up to $30 \%$ of AVNRT, making this an imperfect discriminator between AVRT and AVNRT, and thus a nonspecific indicator for the presence of an accessory AV pathway. ${ }^{11}$ Another confounding factor in detecting the presence or absence of an accessory AV pathway is that accessory AV pathways do not always conduct in an anterograde direction and thus do not demonstrate preexcitation on standard 12-lead ECGs. ${ }^{5}$ These are commonly referred to as "concealed" accessory pathways. Concealed accessory AV pathways may still facilitate arrhythmias or simply lay inactive and/or dormant. Atrioventricular reentry tachycardia in the absence of findings of WPW syndrome on a sinus rhythm ECG is thus also referred to as "concealed" WPW syndrome.

\section{Wolff-Parkinson-White syndrome with atrial fibrillation}

Another concerning feature of AVRT is that it can disorganize into atrial fibrillation, which can have disastrous consequences in patients with accessory AV pathways capable of anterograde conduction. Atrial impulses can reach an accessory pathway at a rate of 300 to 400 times per minute and may result in hemodynamic instability due to rapid rates of ventricular response, far in excess of that allowed by the AV node-His-Purkinje axis. Although accessory pathways typically have longer 
refractory periods than the AV node, and thus should not be capable of pacing the ventricles faster than the AV node, this refractory period can shorten markedly at high rates and allow for a rapider rate of conduction than the $\mathrm{AV}$ node. This manifests as a wide QRS complex tachyarrhythmia with an irregular rate, the differential of which includes WPW syndrome with atrial fibrillation, polymorphic ventricular tachycardia (PVT), and atrial fibrillation with interventricular conduction delay. Distinguishing between these rhythms can be extremely difficult. Having access to a previously recorded ECG in these cases is critical.

Polymorphic ventricular tachycardia tends to exhibit chaotic and haphazard QRS complex voltages as opposed to the other 2 entities and thus is the most likely diagnosis if wide voltage variation in QRS complexes is present. These patients may have a history of alcoholism, poor nutritional status, or excessive diuretic use, all of which predispose to hypomagnesemia and the torsades de pointes variety of PVT. An ECG obtained before the tachycardia may reveal a prolonged QT interval in these individuals. Alternatively, PVT can be sequelae of preexisting ischemic heart disease, congenital

long QT syndrome, or acute coronary syndromes. In most cases, the ECG will suggest the correct diagnosis based on the chaotic electrical nature of this rhythm.

Wolff-Parkinson-White syndrome with AF should be suspected based on clinical history and ECG features. Patients who present with a history of multiple episodes of sudden syncope in the absence of known cardiovascular disease are particularly suspicious. The ECG can demonstrate a variety of findings, with QRS morphologies varying widely depending on the location of the accessory pathway and the relative rate of $\mathrm{AV}$ nodal conduction. The presence of any simultaneous conduction via the $\mathrm{AV}$ nodal pathway may manifest as a fusion beat QRS complex, which can be an additional clue to this rhythm. Patients in this rhythm can deteriorate quickly and require emergent management, either with antiarrhythmic therapy or electrical cardioversion.

\section{Conclusion:}

Wolff-Parkinson-White syndrome is a rare but potentially lethal conduction disturbance that requires specific emergency management. Emergency physicians should thus be intimately familiar with common ECG manifestations. Asymptomatic cases warrant prompt cardiology referral on an outpatient basis, whereas symptomatic cases should be hospitalized in conjunction with electrophysiologic evaluation.

\section{Reference:}

1. Wolff L, Parkinson J, White PD. Bundle-branch block with short P-R interval in healthy young people prone to paroxysmal tachycardia. Am Heart $\mathrm{J}$ 1930;5:685-704.

2. Kent AFS. The structure of cardiac tissues at the auricular ventricular junction: proceedings of the Physiological Society. J Physiol (Lond) 1913;47:17-19.

3. M Holzman and D Scherf, Uber Elektrokardiogramme met verkurzter vorhof-kammer distanz und positiven $\mathrm{P}$ zachen. Ztschr Klin Med 1932; 121:404-23.

4. Ohnell RF. Pre-excitation, a cardiac abnormality. Acta Med Scand 1944; 152:1-167FC.

5. AI-Khatib SM, Pritchett ELC. Clinical features of Wolff-Parkinson-White syndrome. Am Heart J 1999;138:403-13.

6. Wolff L, Parkinson J, White PD. Bundle-branch block with short P-R interval in healthy young people prone to paroxismal tachycardia. Am Heart J 1.930;5:685-704.

7. Scheinman M. History of Wolf- Parkmson-White syndrome. Pace 2005;28:152-6.

8. Plumb V. Catheter ablation of accessory pathways of the Wolff-Parkinson-White syndrome and its variants. Prog in Cardiovasc Dis 1.995;37:295-306.

9. ACC/AHA/ESC guidelines for the management of patients with supraventricular arrhythmias-executive summary. European heart J 2003;24:1857-97.

10. Massumi RA. Familial Wolff-Parkinson-White syndrome with cardiomyopathy. Am J Med 1967;43:951-55.

11. Tortoriello, et al. Frequency of recurrence among infants with supraventricular tachycardia and comparison of recurrence rates among those with and without preexcitation and among those with and without response to digoxin and/or propranolol therapy. Am J Cardiol 2003;92:1045-9. 